\title{
Le quartier épiscopal, campagne 2010, Byllis
} (Albanie)

Nicolas Beaudry et Pascale Chevalier et Skënder Muçaj

\section{Q OpenEdition \\ 1 Journals}

Édition électronique

URL : https://journals.openedition.org/cem/11925

DOI : $10.4000 /$ cem. 11925

ISSN : 1954-3093

\section{Éditeur}

Centre d'études médiévales Saint-Germain d'Auxerre

\section{Édition imprimée}

Pagination : 91-95

ISSN : 1623-5770

\section{Référence électronique}

Nicolas Beaudry et Pascale Chevalier et Skënder Muçaj, «Le quartier épiscopal, campagne 2010, Byllis (Albanie) », Bulletin du centre d'études médiévales d'Auxerre / BUCEMA [En ligne], 15 | 2011, mis en ligne le 01 septembre 2011, consulté le 22 septembre 2022. URL : http://journals.openedition.org/ cem/11925; DOI : https://doi.org/10.4000/cem.11925

Ce document a été généré automatiquement le 22 septembre 2022.

\section{(c) (i) (2) (2)}

Creative Commons - Attribution - Pas d'Utilisation Commerciale - Partage dans les Mêmes Conditions 4.0 International - CC BY-NC-SA 4.0

https://creativecommons.org/licenses/by-nc-sa/4.0/ 


\title{
Le quartier épiscopal, campagne 2010, Byllis (Albanie)
}

\author{
Nicolas Beaudry et Pascale Chevalier et Skënder Muçaj
}

1 La douzième campagne sur le site de Byllis a occupé deux semaines, du 16 au 30 mai 2010․ Rappelons que ces travaux s'effectuent sous l'égide de l'ÉfA et du MAE, qui les financent à parité, avec un apport aujourd'hui presque équivalent du Fond québécois pour la recherche scientifique et culturelle et de l'université du Québec à Rimouski, en collaboration avec l'Institut archéologique de Tirana et le Parc archéologique de Byllis ${ }^{2}$.

2 La mission de 2010 avait pourseul objectif de terminer la fouille du quartier épiscopal. On a parallèlement poursuivi l'étude du matériel découvert en fouille (céramique, verre, os, etc.), ainsi que les flottations et tris carpologiques. P. Chevalier et M. WurchKozelj ont travaillé quelques jours ensemble aux publications Byllis I et II. À la demande du Parc archéologique de Byllis, M. Wurch-Kozelj et T. Kozelj ont brièvement étudié le four à chaux protobyzantin situé au pied du théâtre antique. Enfin, une reconnaissance pédestre rapide leur a permis de reconnaître un site d'extraction de statues dans les carrières de la ville.

3 Après une première approche par S. Muçaj, en 1987-1989, nous avons repris, à partir de 2004, l'étude du secteur urbain dépendant de l'évêché, mais coupé du domaine liturgique et consacré à l'habitat et à des activités économiques variées. La configuration générale du quartier, qui s'étend au sud-est de la cathédrale (fig. 1), a été évoquée à maintes reprises dans les chroniques précédentes ${ }^{3}$, il nous semble peu utile de nous y attarder à nouveau. Rappelons simplement que, dans son dernier état bâti, du $\mathrm{VI}^{\mathrm{e}}$ siècle de notre ère, il est traversé par deux axes perpendiculaires. À leur croisement, la cour L, créée par l'arasement de structures antérieures, desservait des espaces à vocations économique et résidentielle, dont certains ont été examinés plus avant en 2010 à la faveur de quatre sondages. 


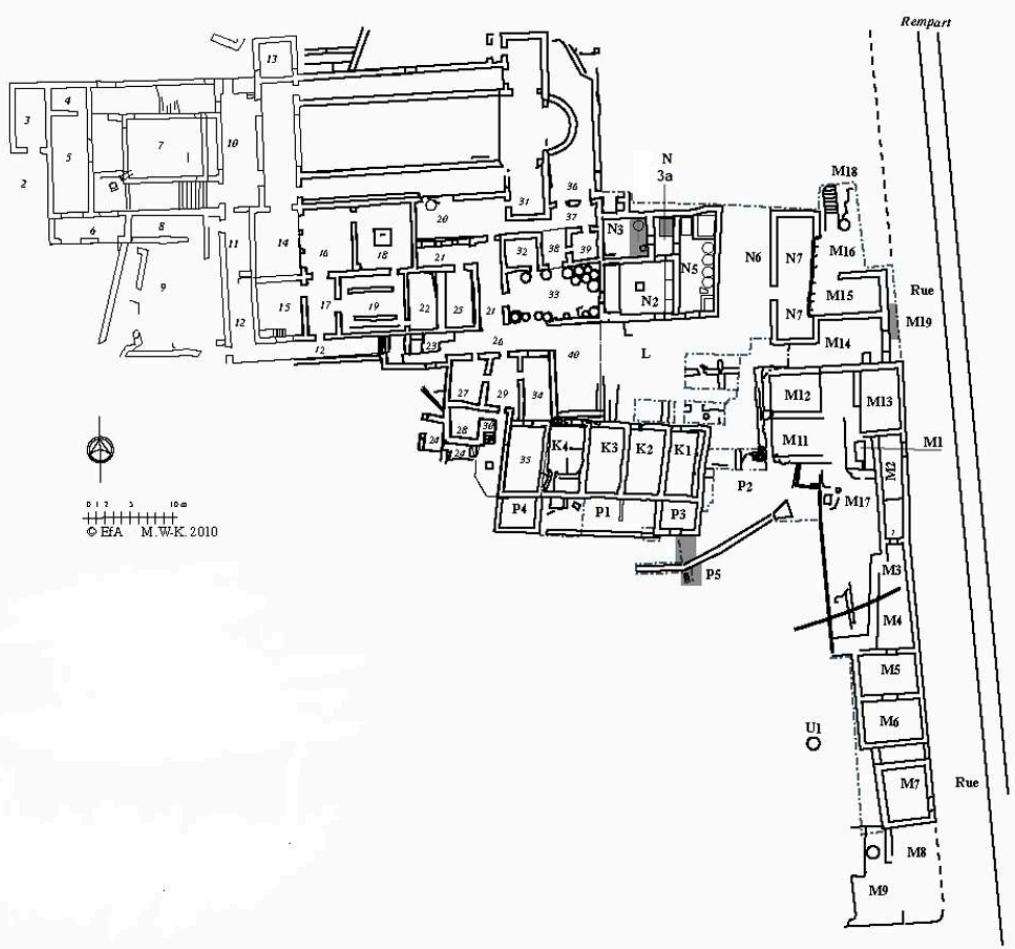

Fig. 1 - Byllis, plan schématique de la cathédrale et du quartier épiscopal : implantation des fouilles et nettoyages en 2010 (dessin M. Wurch-Kozelj).

4 La campagne 2010 étant très courte, ses objectifs se sont en effet limités à la poursuite et la fin d'opérations entreprises en 2009 dans l'installation vinicole (pièces N3, N3A) et en façade méridionale du bâtiment K-P (espace P2), ainsi qu'à l'extension d'un sondage ouvert en 2006 en façade du quartier et dans l'emprise du cardo longeant le rempart protobyzantin. On a aussi continué l'étude et la conservation des structures exposées dans ce secteur depuis 2000.

5 Au sud-est du bâtiment K-P, l'axe de circulation nord-sud traversant la cour est fermé par un mur nord-est - sud-ouest, qui associe des moellons à de gros parpaings et fait un coude vers l'ouest à la hauteur de P3, ménageant un espace de circulation devant la façade du bâtiment K-P. Son tracé suggérait un mur de clôture, délimitant au sud le domaine épiscopal. Une tranchée avait été ouverte en 2009 entre P3 et le mur coudé, livrant un pas-de-porte à l'entrée de P3.

6 Le sondage P2B a été étendu en 2010, d'abord vers l'est puis vers le sud, au-delà du mur coudé. À l'est, un mince sol d'argile fine - dernier niveau de circulation avant l'abandon de P3 et l'effondrement de sa toiture - couvrait le pas-de-porte et l'arasement d'un mur nord-sud, qui porte les restes d'un second mur maçonné, plus étroit, partageant son parement occidental et appartenant à une phase intermédiaire. Au sud, le mur coudé s'appuie sur un mur ouest-est, arasé à la même hauteur que le mur nord-sud, s'infléchissant vers le nord-est à la rencontre des deux murs antérieurs (fig. 2). Sa relation avec le bâtiment K-P reste incertaine, mais il n'est pas exclu qu'ils aient été contemporains, comme le suggère son tracé. 


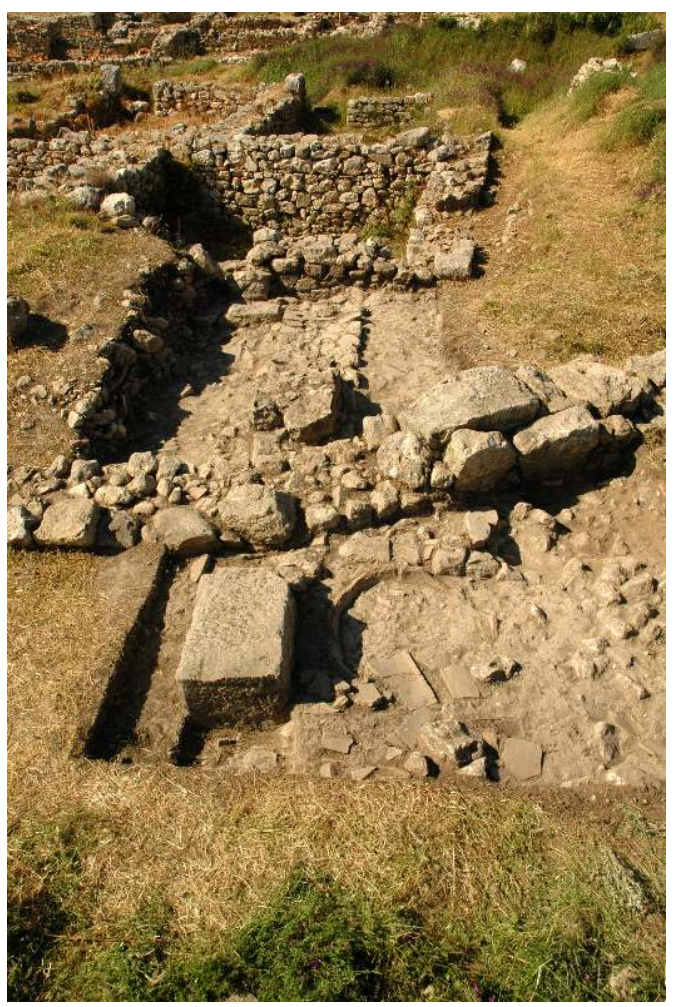

Fig. 2 - Byllis, pièce P3 et sondage P2B, vus vers l'est (cl. N. Beaudry).

7 L'alignement du mur nord-sud est celui du cadastre hellénistique. Il se prolonge vers le sud, au-delà du mur coudé, jusqu'à un jambage de porte. Au nord, il a été coupé par la tranchée de fondation du mur méridional de $\mathrm{P} 3$, mais il se prolonge sous les pièces $\mathrm{P} 3$ et $\mathrm{K} 1$, puis dans des vestiges antérieurs au $\mathrm{VI}^{\mathrm{e}}$ siècle, sous la cour L. Le mur est-ouest appartient à la même phase et pourrait avoir été lié au mur nord-sud. Il porte, en partie, le mur coudé sur une courte distance, au-delà de laquelle il paraît avoir été démonté. Dans l'angle déterminé par ces deux murs, un pithos a été arasé à un niveau comparable à celui des murs. Ces vestiges, peu profonds, laissent entrevoir le potentiel archéologique du plateau qui s'étend au sud du bâtiment K-P, pour la période qui précède le remaniement du quartier et l'expansion de l'évêché dans le courant du $\mathrm{VI}^{\mathrm{e}}$ siècle.

8 Le bâtiment $\mathrm{N}$ prolonge vers l'est la façade du groupe cathédral sur la cour L, avec quatre espaces intérieurs : une salle orientale N5, accessible à partir de la cour ; au sudouest, un cellier N2, qui ouvre sur le magasin (33) ; une pièce nord-ouest N3 ; un petit espace N3A, qui permet la communication entre les trois pièces. N5 s'avère être un chai, comprenant quatre pithoi et deux fouloirs: le premier, dans l'angle sud-est, et, un second, dans l'angle nord-est, qui se déversait vers l'ouest dans un bassin de recueil et de macération, remplissant à son tour, à travers le mur de refend, une cuve de fermentation en N3A. La fouille de N3A a en effet livré une profonde cuve, qui recevait, par un conduit formé d'imbrices traversant le mur mitoyen, le moût décanté dans le bassin de recueil de N5. Au sud de cette cuve, la couche de destruction couvrait un sol noirâtre. Un reliquat de la couche de destruction, au pied de la porte de N3, a été fouillé en 2010 en phase avec celui de la pièce voisine; cette opération a révélé un seuil maçonné, comparable à celui de la porte qui permet la communication entre N3A et N2. La maçonnerie de pierre et de briques des parois de la cuve de fermentation forme une 
margelle saillante adossée, à l'est, au mur mitoyen avec N5, et, au nord, au mur extérieur. Sur les côtés nord, ouest et sud, des briques liées au mortier forment une feuillure qui maintenait un couvercle de bois. Les parois intérieures et le rebord de la cuve sont enduits d'un mortier hydraulique rechapé au moins deux fois. Elle est munie d'un escalier de deux degrés dans son angle sud-ouest et son fond est aménagé d'une cupule hémisphérique de plan elliptique.

La fouille des niveaux de destruction de la pièce N3 a été complétée à l'est en 2010. La couche de tuiles de toiture était particulièrement dense dans les parties centrale et septentrionale du sondage. Cette opération a exposé le mortier inscrit, aperçu en 2009 ; elle a, en outre, livré,in situ, un troisième pithos, dont les parois intérieures sont enduites de bitume, ainsi que les fragments d'un quatrième, concentrés contre le mur sud. Les derniers niveaux de destruction et d'abandon ont révélé des céramiques et un mobilier métallique comprenant quelques clous et des éléments d'huisserie.

En 2006, une tranchée en façade orientale du quartier a exposé un mortier très dégradé, établi à même le rocher au pied de la façade de la pièce M15. Il pouvait s'agir des vestiges d'un aménagement en front de rue ou, comme le suggère l'alignement des façades au sud du portail M14, d'un aménagement intérieur, qui aurait précédé un déplacement vers l'ouest de la façade de M15, à la suite de l'empiétement du rempart de Viktôrinos sur le cardo. Cette tranchée a été nettoyée et étendue en 2010 pour vérifier l'état des vestiges et tester ces hypothèses. Le sondage a livré un arasement horizontal du rocher, qui se serait bien prêté à l'aménagement, cependant aucun négatif dans le mortier ni aucune trace rupestre ne permet de conclusion.

\section{NOTES}

1. L'équipe se composait ainsi :

- du côté albanais : Skënder Muçaj, Institut d'études albanologiques, Institut archéologique de Tirana, section Antiquité tardive-Moyen Âge, université de Tirana (chef de mission); Skënder Bushi, dessinateur; Syela Xhyeri, archéologue (IAT); Ornela Durmishaj, archéologue (Parc archéologique de Byllis); Mariglen Meshini, étudiant (université de Tirana); Restaurations et consolidations : Admir Xhelaj, restaurateur (Parc archéologique d'Apollonia);

- du côté franco-canadien :Pascale Chevalier, université Blaise-Pascal Clermont-Ferrand 2, UMR 5594-ARTeHIS, CNRS (chef de mission); Nicolas Beaudry, université du Québec à Rimouski (responsable de l'étude du quartier épiscopal) ; Tony Koželj et Manuela Wurch-Koželj, architectes (École française d'Athènes) ; Catherine Dupont, étudiante (université de Montréal et de Lille 3) ; Julie Viriot, étudiante (UBP) ; Julien Bruyère et Sylvain Grosfilley, étudiants (université de SaintÉtienne) ; Chantal Gagné, étudiante (UQAR) et Nadia Saint-Luc.

Des analyses ont été réalisées en France et au Canada, par Stéphane Büttner, géoarchéologue (Centre d'études médiévales, UMR 5594) ; Manon Savard et C. Gagné, paléo-environnementalistes (UQAR). Enfin, P. Chevalier et M.-P. Raynaud, avec la collaboration ponctuelle de M. Wurch-Kozelj et de Jean-Pierre Sodini, ont achevé la révision finale du volume Byllis I, lors de réunions successives à Paris en mars, avril, juin et septembre. Le manuscrit définitif a été reçu à l'ÉfA le 
20 septembre 2010, il paraîtra prochainement dans la collection RAFAL des Écoles françaises de Rome et d'Athènes.

2. Bucema, 12 (2008), p. 63-68 [http://cem.revues.org/index6512.html]; Bucema, 13 (2009), p. 73-76 [http://cem.revues.org/index11233.html] et Bucema, 14 (2010), p.57-60 [http:// cem.revues.org/index11539.html].

3. Mission 2005: Bulletin de correspondance hellénique $[B C H], 130 / 2$ (2006), p. 836-840; mission 2006 : BCH, 131/2 (2007), p. 1098-1113 ; mission 2007 : BCH, 132/2 (2008), p. 925-936. Ou, encore, en dernier lieu, Bucema, 14 (2010), p. 57-60 [http://cem.revues.org/index11539.html].

INDEX

Mots-clés : quartier épiscopal

Index géographique : Albanie/Byllis 